\title{
Interférences
}

Ars scribendi

$8 \mid 2015$

L'exil au miroir de la Correspondance de Cicéron

\section{De l'exil à la domus : les problèmes de droit liés à l'exil de Cicéron}

\section{Michèle Ducos}

\section{(2) OpenEdition \\ 1 Journals}

Édition électronique

URL : http://journals.openedition.org/interferences/5453

DOI : 10.4000/interferences.5453

ISSN : $1777-5485$

Éditeur

HiSoMA - Histoire et sources des Mondes antiques

Référence électronique

Michèle Ducos, «De l'exil à la domus : les problèmes de droit liés à l'exil de Cicéron », Interférences [En ligne], 8 | 2015, mis en ligne le 11 décembre 2014, consulté le 10 décembre 2020. URL : http:// journals.openedition.org/interferences/5453; DOI : https://doi.org/10.4000/interferences.5453

Ce document a été généré automatiquement le 10 décembre 2020.

Tous droits réservés 


\title{
De l'exil à la domus : les problèmes de droit liés à l'exil de Cicéron
}

\author{
Michèle Ducos
}

1 Les lettres de Cicéron contiennent de nombreuses références au droit public et privé : allusions rapides ou réflexions plus poussées sur quelques cas complexes révèlent l'intérêt manifeste de l'écrivain pour ces questions. La période de l'exil où Cicéron met en scène son désespoir semble devoir être mise à part et constituer une exception. Pourtant, on trouve aussi dans ces lettres une attention précise aux questions juridiques liées à la situation personnelle du consulaire et à ses espoirs de retour. Adressées à des intimes qui n'ignorent rien des causes de l'exil ni du contexte politique et juridique, ces lettres sont souvent allusives : Cicéron donne des commentaires précis, mais partiels. Souvent, le recours à d'autres sources ou à des discours postérieurs s'impose. Cet ensemble permet de mieux comprendre l'exil de l'orateur et les conditions de son retour.

2 Sous la République, à la différence du Principat, l'exil n'est pas une peine. Un passage souvent cité du Pro Caecina en précise très nettement la signification : dans aucune loi, souligne l'orateur, on ne trouvera qu'un crime ait été puni d'exil (Cic., Caec. 100) :

Exsilium enim non supplicium est, sed perfugium portusque supplici. Nam quia uolunt poenam aliquam subterfugere aut calamitatem, eo solum uertunt, hoc est sedem ac locum mutant. Itaque nulla in lege nostra reperietur, <ut> apud ceteras ciuitates, maleficium ullum exsilio esse multatum; sed cum homines uincula, neces ignominiasque uitant, quae sunt legibus constitutae, confugiunt quasi ad aram in exsilium.

L'exil n'est pas un châtiment, mais un refuge et un port pour un suppliant. En effet, quand on veut échapper à une peine ou à un désastre, on change de sol, c'est-à-dire que l'on transforme sa résidence et son lieu de séjour. C'est pourquoi dans aucune de nos lois, on ne trouvera qu'un méfait ait été puni de l'exil, comme cela se passe dans les autres cités, mais quand les hommes évitent les chaînes, la mort et l'infamie, qui sont établies par les lois, ils fuient en exil comme auprès d'un autel.

Ainsi l'exil est clairement présenté comme un choix délibéré, que marque le verbe uolunt. Cicéron l'oppose aux peines établies par le droit, pour montrer nettement que ce départ en exil échappe au droit pénal. De fait, à Rome, tout accusé qui risque d'encourir une condamnation à la peine capitale ou à une forte amende a la possibilité de quitter 
Rome et de s'installer dans une autre cité ${ }^{1}$. Tel fut le cas de Verrès ou de Milon. L'exil permet alors d'échapper aux sanctions, d'où le lexique du refuge et de la protection: perfugium, portus, ara.

En mars 58 , la conduite de Cicéron semble presque identique ${ }^{2}$ : il quitte Rome à la suite de la menace que représente pour lui la rogatio présentée par Clodius, alors tribun de la plèbe. Mais cet éloignement qui devait être de courte durée ${ }^{3}$ dura plus d'une année. En effet, Clodius avait déposé un projet de loi concernant «quiconque aurait tué ou fait tuer un citoyen romain sans jugement ». Était donc sanctionnée par la lex Clodia de capite ciuis Romani la mise à mort d'un citoyen romain sans condamnation préalable ni procès.

5 Le texte de la loi ne nous est pas connu exactement ${ }^{4}$ : selon Velleius Paterculus, "Clodius proposa une loi selon laquelle celui qui aurait fait périr un citoyen romain, qui n'aurait pas été condamné, serait interdit d'eau et de feu ${ }^{5} »$. Dion Cassius indique "que la loi visait en général tous ceux qui mettraient ou avaient mis à mort un citoyen sans jugement ${ }^{6} \%$. Même si son nom n'était pas mentionné expressément, Cicéron était directement visé par cette loi, à cause de l'exécution des complices de Catilina en décembre $63^{7}$. Appien et Plutarque font même allusion à un procès intenté à Cicéron ${ }^{8}$. Les expressions utilisées par les historiens anciens laissent penser que cette loi avait une valeur rétroactive, visant des actes antérieurs à la loi ${ }^{9}$. Mais la rogatio était générale et ne comportait pas d'indication de procédure ni de peine. Elle reprenait clairement la disposition de la lex Sempronia de Caius Gracchus (en 123 av. J.-C.) interdisant de mettre à mort un citoyen romain sans condamnation. Clodius réaffirmait ce principe ancien, sans innovation ${ }^{10}$, mais en l'élargissant à tous ceux qui auraient fait mettre à mort un citoyen ${ }^{11}$.

6 Une fois la loi votée par les comices, le tribun était en mesure de lancer une action en justice contre Cicéron et de le soumettre à un procès infamant, qui aurait sans doute été un iudicium populi, un procès devant les comices : son issue ne faisait aucun doute. C'est précisément ce qui effraya Cicéron; ne rencontrant aucun soutien, écoutant les conseils d'amis (ou de ceux qu'il croyait encore ses amis), il quitta Rome la veille du jour où le plébiscite allait être voté.

7 En fait, comme Cicéron le reconnaît lui-même dans une lettre à Atticus datée du mois d'août 58 , cette loi «ne l'atteignait en rien ${ }^{12} »$. En méditant avec désespoir sur sa condition, en revenant sur le passé, il se livre à une nouvelle lecture de la loi et souligne son erreur de jugement : le texte était général et ne pouvait en rien lui nuire tant qu'on n'avait pas intenté d'action contre lui (nisi nominatim mecum agi coeptum esset). Il a même été suggéré que le risque d'un procès (y compris un procès comitial) et, par conséquent, celui d'une condamnation était minime ${ }^{13}$; d'autres historiens sont au contraire d'avis que la menace était réelle, car les sénateurs ne pouvaient pas s'opposer à une décision du peuple par un sénatus-consulte qui aurait protégé Cicéron ${ }^{14}$.

8 Cicéron quitta donc Rome; il resta d'abord en Italie, à une courte distance de la Ville, jusqu'au début du mois d'avril ${ }^{15}$ : la distance le protégeait contre toute contrainte tribunicienne, car le pouvoir des tribuns s'arrêtait à la première borne milliaire.

9 Après le départ de Cicéron ${ }^{16}$, Clodius déposa une seconde rogatio, appelée souvent lex de exilio Ciceronis. Il n'y a donc pas eu de procès ni, en l'absence de l'accusé, de reconnaissance d'un état d'exil entraînant une aqua et igni interdictio. Tel était l'usage, 
comme le montre par exemple le procès de M. Postumius Pyrgensis accusé par les tribuns (Liv., 25, 4, 9) :

Tribuni plebem rogauerunt plebesque ita sciuit, si M. Postumius ante kalendas Maias non prodisset citatusque eo die non respondisset neque excusatus esset, uideri eum in exsilio esse bonaque eius uenire, ipsi aqua et igni placere interdici.

les tribuns demandèrent à la plèbe et la plèbe décida que si M. Postumius ne s'était pas présenté avant les calendes de mai, si ce jour-là, sans s'être fait excuser, il n'avait pas répondu à la convocation, on le considérerait comme exilé et il serait décidé que ses biens seraient vendus et qu'on lui interdirait l'eau et le feu.

Le concilium plebis enregistre donc le fait que l'accusé ne s'est pas présenté et qu'il s'est exilé. La conséquence en est l'aqua et igni interdictio.

11 Clodius a voulu, lui aussi, transformer un départ volontaire en exil : donner à une situation de fait un caractère juridique ${ }^{17}$, c'est-à-dire en faire un exilium iustum fondé sur une décision du peuple, en l'occurrence un plébiscite. Mais Clodius a éliminé la phase du procès et de la condamnation et sa loi n'instituait aucune procédure judiciaire contre l'orateur ${ }^{18}$. À son retour, Cicéron peut ainsi expliquer, dans les discours qu'il prononce alors, qu'il n'a pas été condamné à la suite d'un procès : «moi, qui n'ai jamais reçu d'assignation à comparaître, qui n'ai pas été accusé, qui n'ai jamais été cité par un tribun de la plèbe $[. . .]^{19}$ ». Le lexique qu'il utilise dans les lettres et dans les discours post reditum évite les termes évoquant trop précisément l'exil ${ }^{20}$ et Cicéron rappelle qu'il est integer et indemnatus ${ }^{21}$; en même temps, il dénonce l'erreur de Clodius. Pour le tribun, ce départ assurément était un aveu de culpabilité ${ }^{22}$ : une action judiciaire ne semblait sans doute pas nécessaire, mais il fallait confirmer cette culpabilité par une loi et empêcher les sénateurs de manifester à Cicéron leur soutien ${ }^{23}$.

Le projet de loi affiché une première fois fut modifié et finalement voté fin avril, comme le montrent les premières lettres de l'exil où il est fait mention d'une rogatio correcta $^{24}$. Une telle pratique, qui peut nous paraître surprenante, n'est pas inconnue à Rome et il en existe d'autres exemples ${ }^{25}$. Selon la loi, Cicéron est contraint de se tenir éloigné de cinq cents milles ${ }^{26}$ des côtes de l'Italie : la zone interdite a été agrandie dans la nouvelle rédaction. Cette transformation peut témoigner de la maladresse d'une rédaction initiale trop hâtive ${ }^{27}$; elle pourrait aussi être l'écho de débats dans une contio ${ }^{28}$. Le nouvel affichage eut lieu en avril et le vote fin avril.

13 La loi comportait de nombreux articles que nous ne connaissons que très imparfaitement à travers les lettres de Cicéron et les discours prononcés après le retour d'exil.

Elle comprenait à coup sûr la confirmation de l'exil et l'interdiction de l'eau et du feu ; s'y ajoutaient la confiscation des biens de Cicéron ${ }^{29}$, la confiscation des propriétés et la destruction des constructions (dont la maison de Cicéron sur le Palatin et ses villas de Tusculum et de Formies). Enfin, la loi était accompagnée de très nombreuses clauses de sauvegarde figurant dans la sanctio. Ce texte, sans doute assez long, traitait donc de questions multiples, certes concernant toutes l'exil de Cicéron, mais d'une grande variété : la loi constitue ainsi pour Cicéron une lex satura (ou per saturam), une loi mêlant des articles divers ${ }^{30}$, un type de loi qui était interdit à Rome depuis la lex Caecilia Didia de 98 av. J.-C. Il s'agissait ainsi d'une première illégalité.

La loi de Clodius décidait de l'exil de Cicéron et, plus exactement, lui interdisait l'eau et le feu : il était interdit de lui donner l'hospitalité et de le recevoir sous son toit. Une telle interdiction empêchait le retour de l'exilé en territoire romain, puisque personne 
ne pouvait le recevoir, et le confirmait dans son état d'exilé. Dans la loi, des peines étaient prévues pour ceux qui lui donneraient asile: ils pouvaient être mis à mort impunément ${ }^{31}$. De fait, Cicéron s'abstient de s'arrêter chez certains de ses amis, comme Sicca ; il reste dans les environs de Brindes chez M. Lénius Flaccus ${ }^{32}$.

Selon Cicéron, la loi était mal rédigée. Clodius, ou Sex. Clodius, le rédacteur de la loi, avait employé le subjonctif parfait au lieu du subjonctif présent marquant l'avenir ${ }^{33}$ : interdictum sit alors qu'on attendrait interdicatur. L'aqua et igni interdictio ne comportait pas de date d'entrée en vigueur et l'interdiction était présentée comme une situation déjà existante qu'il fallait confirmer. Pour sa part, l'orateur n'a cessé de railler le legum scriptor peritus et callidus, auteur de cette formule car, à ses yeux, il s'agissait en quelque sorte de voter pour décider de ce qui avait déjà été fait : il souligne l'incohérence qui consiste à «proposer au peuple de sanctionner par quelque formule ou de confirmer par son vote que ce qui n'a pas été fait ait été fait ${ }^{34} »$.

Le départ de Cicéron avant le vote de la lex de capite ciuis Romani ne permettait pas de l'inculper en s'appuyant sur cette loi. Mais, comme le marquent les citations de Cicéron, la loi était précédée de deux clauses, introduites par quod, qui exprimaient les raisons de l'interdiction ${ }^{35}$. Le premier de ces motifs était évidemment l'exécution de citoyens sans jugement ${ }^{36}$; le second était plus inattendu: Cicéron était coupable d'avoir falsifié un sénatus-consulte, selon le De domo ${ }^{37}$; le sénatus-consulte était celui qui avait permis l'exécution des complices de Catilina le 5 décembre 63. Une telle affirmation était surprenante : Cicéron avait eu recours à des sténographes et avait fait faire un compte-rendu minutieux de la séance, aussitôt diffusé ${ }^{38}$. Mais avec cette accusation de faux, le sénat n'était plus responsable collectivement de l'exécution. La faute était rejetée sur le seul Cicéron, qui avait paré une exécution illégale du voile de la légalité ${ }^{39}$. Il tombait alors sous le coup de la loi et pouvait être accusé seul.

Cicéron n'a cessé d'attaquer cette loi et d'en dénoncer l'illégalité. La question n'est pas limitée aux discours liés au retour d'exil; elle parcourt son œuvre. Liée d'abord à sa situation d'exilé, elle occupe progressivement une place importante dans sa réflexion politique et juridique. Selon lui, la loi de Clodius est ce qu'il appelle priuilegium, c'està-dire une mesure dirigée nommément contre un particulier. Ce terme appartient depuis longtemps au lexique juridique: il figure chez Aulu-Gelle, chez Festus, chez Ateius Capito, un juriste du début du principat ${ }^{40}$. Selon l'orateur, de telles décisions sont contraires au principe d'égalité qui fonde la loi ${ }^{41}$. En effet, la loi ne s'applique pas seulement à un citoyen, mais à tous, et les traite de façon identique. Cicéron rappelle à plusieurs reprises l'ancienneté de cet interdit remontant aux temps les plus anciens de la cité et aux lois les plus vénérables : les lois sacrées et la loi des XII Tables. C'est bien ce qu'explique le De domo (Cic., Dom. 43) :

Quo iure, quo more, quo exemplo legem nominatim de capite ciuis indemnati tulisti? Vetant leges sacratae, uetant XII tabulae leges priuatis hominibus inrogari ; id est enim priuilegium. Nemo umquam tulit; nihil est crudelius, nihil perniciosius, nihil quod minus haec ciuitas ferre possit.

Par quel droit, par quel usage, par quel précédent as-tu présenté une loi qui visait nommément la tête d'un citoyen qui n'avait pas été condamné ? Il est interdit par les lois sacrées, il est interdit par les XII Tables de proposer des lois contre des particuliers : c'est en effet un 'privilège'; rien n'est plus cruel, rien n'est plus dangereux, rien n'est plus intolérable pour notre cité. ciuis indemnati ferre; leges priuatis hominibus inrogare. L'emploi de l'adverbe nominatim, 
l'insistance sur les particuliers qui sont visés mettent en évidence le caractère dangereux de la loi et en accentuent l'illégalité. Dans les lignes suivantes, cette décision est mise en parallèle avec les proscriptions de Sylla, qui étaient aussi décidées sans jugement et dirigées contre des particuliers mentionnés nommément. De plus, avec l'adjectif indemnatus ou l'expression sine iudicio, est soulignée l'illégalité de cette décision prononcée sans jugement. Cicéron retourne ainsi habilement contre Clodius l'accusation que ce dernier avait portée contre lui.

Ainsi Clodius s'est opposé aux lois fondamentales de la cité et il a choisi de condamner un citoyen sans jugement. C'est sans doute cet argument que comptait utiliser Q. Terentius Culleo pour faire invalider la loi par le sénat ${ }^{42}$. C'est aussi ce que soulignait L. Aurelius Cotta ${ }^{43}$ : «rien absolument n'avait été décidé à notre sujet»; de fait, les priuilegia sont contraires aux lois romaines et les comices tributes n'étaient pas compétents pour une affaire capitale : une loi n'aurait donc pas été nécessaire. Malgré ces raisons solides, Cicéron avait une nette préférence pour une abrogation en règle par une loi comitiale votée par les comices centuriates, car une telle décision ne pouvait être facilement remise en cause ${ }^{44}$.

21 Pourtant, la loi de Clodius était entourée de multiples clauses n'interdisant pas seulement son abrogation, mais interdisant même d'en parler ; cet article avait ainsi été affiché à la porte du sénat pour rappeler aux sénateurs cette interdiction ${ }^{45}$. Une autre clause de sauvegarde extrêmement minutieuse figurait dans la partie finale de la loi. C'est ce que montre clairement la rogatio des huit tribuns, promulguée fin octobre 58, qui devait permettre le retour de Cicéron. Dans une lettre de fin novembre 58 adressée à Atticus, Cicéron se livre à une analyse détaillée de ce projet. Il donne un commentaire développé et minutieux des articles de la loi et des formules utilisées par leurs auteurs; cette analyse ne surprend pas, car l'exilé examine avec une attention scrupuleuse les projets concernant son retour et les conséquences juridiques de telles propositions.

Le premier article concernait le retour de Cicéron, mais il ne satisfait pas ce dernier, car les restitutions proposées (droit de cité et place au sénat) sont insuffisantes à ses yeux ${ }^{46}$ et le texte est jugé scriptum incaute ( rédigé sans précaution »). Le second article contenait la clause d'impunité présente dans la plupart des lois ${ }^{47}$ : elle a pour but d'empêcher toute procédure et toute peine pour un acte accompli contrairement à une loi pour une autre loi ; il ne s'agit pas d'actes contraires à une loi ancienne, qu'une loi nouvelle autorise ${ }^{48}$, mais d'actes faits pour « aboutir à la présente loi » (eius legis ergo). La disposition concerne le rogator de la nouvelle loi et le protège. Et de fait, selon Cicéron, « on ne tient pas compte des sanctions quand on abroge une loi ${ }^{49}$ ».

23 Le troisième article était plus complexe dans sa formulation et même inhabituel, car « il n'existe dans aucune loi un article de ce type ${ }^{50}$ ». La rédaction en était très minutieuse (Cic., Att. 3, 23, 3) :

Si quid in rogatione scriptum est quod per leges pl.ue sc., promulgare, abrogare, derogare, obrogare sine fraude sua non liceat, non licuerit, quodue ei qui promulgauit, abrogauit, derogauit, obrogauit, ob eam rem poenae multaeue sit, e.h. l. n. $r$.

Si est rédigé dans cette proposition un article qu'il ne soit pas ou qu'il n'ait pas été licite par les lois ou les plébiscites de promulguer, d'abroger totalement, d'abroger partiellement, d'abroger tacitement sans dommage pour soi, et qui pour celui qui l'a promulgué, abrogé totalement, abrogé partiellement, abrogé partiellement entraîne peine ou amende, rien de cela n'est proposé par la présente loi ${ }^{51}$.

On notera l'extrême précision du vocabulaire : abrogare, derogare, obrogare; même si cette énumération minutieuse ne surprend pas dans une rogatio datant de la fin de la 
République $^{52}$, sont envisagées toutes les possibilités de modification apportées à une loi : les tribuns ont été particulièrement attentifs à la formulation d'une clause, qui constituait une innovation. Il était par là affirmé que la rogatio ne contenait aucune proposition d'abrogation d'une loi dont l'abrogation serait interdite ${ }^{53}$. Elle protégeait les tribuns en cas d'échec, mais une telle disposition vidait en somme l'article de tout contenu; il n'est donc pas surprenant qu'elle satisfasse pleinement Clodius qui déclare que toute rogatio postérieure, concernant Cicéron, devra contenir cet article; elle suscite les soupçons de Cicéron qui y voit une intention malveillante dirigée contre lui. Ce sentiment est accru par la conviction que la clause est inutile, car ces tribuns n'étaient pas liés par la loi de leur collège ${ }^{54}$. Il espère d'ailleurs que d'autres magistrats défendront mieux sa cause. On peut alors s'étonner du choix des huit tribuns de 58 : mais l'article les protégeait si la proposition échouait ${ }^{55}$ et les mettait donc à l'abri de poursuites. C'est une mesure prudente et habile: Atticus lui-même avait souligné l'utilité de cette promulgation dans une lettre adressée à son ami, car l'affichage d'une rogatio permettait de poser clairement devant le peuple la question du rappel de Cicéron.

De sénatus-consultes en rogationes, le retour de Cicéron fut acquis par une loi votée par les comices centuriates en août 57. C'est bien l'abrogation qu'il attendait. Après son retour triomphal, après les remerciements adressés au sénat et au peuple romain, Cicéron cherche à retrouver son rang et sa dignitas. N'ayant pas subi de condamnation infamante, n'ayant pas renoncé à sa citoyenneté, il est toujours un citoyen romain. Il entend aussi récupérer ses biens : les lettres adressées à Atticus et à Terentia montrent clairement l'importance de cette préoccupation affirmée à plusieurs reprises : « de tous mes biens il n'est rien que j'aime mieux que ma demeure ${ }^{56}$ ", et il déclarait aussi à Terentia : "pour ce que tu m'écris de ma maison, c'est-à-dire de son emplacement, je ne me croirai rétabli dans mes droits, que si elle m'est restituée ${ }^{57} »$. Ce souci peut paraître étonnant; mais il n'est pas surprenant, car, selon Cicéron, la demeure d'un consulaire doit être digne de lui et en accord avec son rang ${ }^{58}$.

Sa restitution fait l'objet d'allusions récurrentes : en effet, la maison de Cicéron sur le Palatin n'avait pas seulement été confisquée ; elle avait été pillée et incendiée le soir de son départ; une clause de la lex de exilio avait sans doute confié à Clodius le soin de contrôler la destruction et la construction du monumentum ${ }^{59}$. Le tribun avait élevé un portique sur le terrain, consacré une partie du terrain et construit un sanctuaire de la Liberté. Pour récupérer le terrain, il fallait donc lui ôter son caractère sacré, ce qui supposait une décision des pontifes.

En septembre 57, après son retour, Cicéron explique à Atticus qu'il attend une réponse des pontifes ${ }^{60}$ puis, dans la lettre suivante, en octobre 57 , il évoque le procès où il parla devant les pontifes avec éloquence et émotion la veille des calendes d'octobre ${ }^{61}$. S'adressant à Atticus qui n'ignore rien des faits passés ni de la situation créée par Clodius, il reste allusif et se borne à évoquer son plaidoyer. L'issue favorable s'exprime dans la décision du collège pontifical annulant la consécration et permettant la restitution du terrain; Cicéron cite les termes mêmes du décret pour faire nettement apparaître l'illégalité de la consécration opérée par Clodius.

C'était précisément l'un des arguments du De domo, prononcé devant les pontifes. Dans ce plaidoyer, la question même de la maison et de la consécration n'occupe qu'une faible place, mais elle constitue l'une des multiples illégalités qui sont l'œuvre de Clodius. Le tribun s'était attribué à lui-même la charge de contrôler la destruction et les 
constructions ${ }^{62}$. De plus, le tribun n'a pas tenu compte de la lex Papiria interdisant de consacrer un édifice sans un vote du peuple ${ }^{63}$ : c'est d'ailleurs l'argument que reprennent les pontifes dans leur décision. Enfin, le pontife encore inexpérimenté choisi par Clodius n'a pas fait la consécration dans les règles ${ }^{64}$. Ces arguments aboutirent au décret des pontifes mentionné dans la lettre à Atticus; l'enjeu du débat n'était pas uniquement juridique, il était politique : la demeure symbolisait la réussite de Cicéron et son succès comme consul ${ }^{65}$; la destruction et sa disparition la ruine voulue et obtenue par Clodius; l'aedes Libertatis élevé par le tribun proclamait sa victoire.

Les problèmes liés à l'exil de Cicéron sont nombreux, précis et complexes : ils mêlent droit privé, religion et droit public. Nous ne connaissons pas avec précision l'ensemble des mesures législatives qui apparaissent à travers les lettres ou les discours post reditum. Et l'on peut s'interroger sur les déformations qu'a pu leur apporter l'éloquence cicéronienne. Mais cet ensemble montre l'extrême attention portée à ces questions par l'homme politique et le citoyen qu'est Cicéron: il cite les textes législatifs et les commente. De plus, cette situation et les problèmes juridiques qu'elle entraîne ont une influence décisive sur les théories politiques et juridiques qui seront formulées plus tard. En même temps, la politique est toujours présente dans ces débats : ils s'insèrent dans les luttes des factions, l'opposition entre optimates et populares, les tensions entre sénateurs et tribuns. Les uns et les autres ont recours au droit: mais il ne sert pas toujours à protéger, et il peut détruire. S'esquisse aussi l'histoire des années suivantes avec sa violence qui fait oublier le droit.

BIBLIOGRAPHIE

\section{Textes anciens}

Cicero's Letters to Atticus, II, ed. by D. R. Shackleton Bailey, Cambridge Classical Texts and Commentaries 4, Cambridge, 1965.

\section{Tetes modernes}

BERG B. 1997, « Cicero's Palatine Home and Clodius' Shrine of Liberty: Alternative Emblems of the Republic in Cicero's De domo sua ", in C. Deroux (éd.), Studies in Latin Literature and Roman History, VIII, Latomus 239, Bruxelles, p. 122-143.

CRIFò G. 1961, Ricerche sull'« exilium » nel periodo repubblicano, I, Fondazione Guglielmo Castelli 32, Milan, p. 175-184.

Ducos M. 1984, Les Romains et la loi. Recherches sur les rapports de la philosophie grecque et de la tradition romaine à la fin de la République, Coll. d'études anciennes, Paris.

GARCEA A. 2005, Cicerone in esilio. L'epistolario e le passioni, Spudasmata 103, Hildesheim - New York. 
GRIMAL P. 1967, Études de chronologie cicéronienne. Années 58 et 57 av. J.-C., Coll. d'études anciennes, Paris.

GRUEN E. 1974, The Last Generation of the Roman Republic, Berkeley - Londres.

HUMBERT M. 1996, «Les privilèges des XII Tables à Cicéron », in A. Chastagnol, S. Demougin, Cl. Lepelley (éds), Spendidissima civitas. Études d'histoire romaine en hommage à François Jacques, Histoire ancienne et médiévale 40, Paris, p. 151-168.

KELLY G. P. 2006, A History of Exile in the Roman Republic, Cambridge.

MAROUZEAU J. 1959, « Sur deux aspects de la langue du droit », in Droits de l'Antiquité et sociologie juridique. Mélanges offerts à Henri Lévy-Bruhl, Publications de l'Institut de droit romain de l'Université de Paris 17, Paris, p. 435-444.

MOMMSEN Th. 1892, Le droit public romain, I, trad. par P. Fr. Girard, Paris.

MOREAU Ph. 1987, «La Lex Clodia sur le bannissement de Cicéron », Athenaeum 65, p. 465-492.

- 1989, « La rogatio des huit tribuns de 58 av. J.-C. et les clauses de sanctio réglementant

l'abrogation des lois ", Athenaeum 67, p. 151-178.

- 2005, «Sublata priore lege. Le retrait des rogationes comme mode d'amendement aux propositions de loi à la fin de la République ", in P. Sineux (éd.), Le législateur et la loi dans l'Antiquité. Hommage à Françoise Ruzé. Actes du colloque de Caen, 15-17 mai 2003, Caen, p. 201-213.

- 2012, « Exiler Cicéron. La lex Clodia de capite ciuis (58 av. J.-C.) a-t-elle comporté une clause de serment? ", in R. Baudry, S. Destephen (éds), La société romaine et ses élites. Hommages à Élizabeth Deniaux, Paris, p. 35-42.

VENTURINI C. 1996, «I 'privilegia' da Cicerone ai Romanisti », in Processo penale e società politica nella Roma repubblicana, Diritto, Pise, p. 239-286 [= SDHI 56, 1990, p. 155-196].

- 2010, «L'esilio di Cicerone tra diritto e compromesso politico », in C. Russo Ruggieri (éd.), Studi in onore di Antonino Metro, VI, Pubblicazioni della Facoltà di giurisprudenza della Università di Messina 248, Milan, p. 427-446.

\section{NOTES}

1. C'est aussi ce qu'a souligné Polybe (Plb., $6,14,7):$ "Dans un procès capital, quand un accusé va être condammné, la coutume lui donne le droit de s'en aller sans se cacher, en s'infligeant un exil volontaire, tant qu'une seule des tribus qui prononcent la sentence n'a pas encore voté » (trad. R. Weil, CUF, Paris, 1977) ; voir Kelly 2006.

2. La date du départ est très discutée (11 mars ? 19 mars ?). Voir Grimal 1967, p. 31-61.

3. Cic., Q. fr. 1, 4, 4 : saepe triduo summa cum gloria dicebar esse rediturus; cf. Cic., Att. 3, 7, 2.

4. Venturini 2010 souligne le silence et la confusion des sources ; voir aussi Moreau 1987.

5. Vell., 2, 45, 1 : P. Clodius [...] legem in tribunatu tulit qui ciuem Romanum indemnatum interemisset, ei aqua et igni interdiceretur [...]. Mentionnée sous cette forme, la loi semble amalgamer les deux mesures de Clodius ou plutôt se référer à des usages postérieurs à la République, car l'aqua et igni interdictio n'est pas à cette date une peine prévue par la loi (Venturini 2010, p. 432).

6. Dion Cassius, 38, 14, 4, dit très exactement : « en l'absence d'une décision du peuple ».

7. Comme le précisent les auteurs anciens : Vell., 2, 45, 2 : cuius uerbis etsi non nominabatur Cicero, tamen solus petebatur; Dion Cassius, 38, 8, $4:$ «bien qu'elle ne fût pas en apparence dirigée contre lui, puisqu'elle ne comportait même pas son nom [...] était en réalité tout particulièrement 
dirigée contre lui ». En effet, l'exécution des complices de Catilina, le 5 décembre 63, faisait l'objet d'un débat entre optimates et populares, concernant le sénatus-consulte ultime et le rôle du sénat. 8. Plu., Cic. 31, 6 : «telle était l'accusation portée contre Cicéron et c'est pour cela qu'il fut cité en justice »; App., BC 2, 15.

9. Gruen 1974, p. 244-246; Moreau 1987, p. 472 ; Kelly 2006, p. 226. Selon Dion Cassius, 38, 17, 2, César avait déclaré dans une contio «qu'il n'estimait pas approprié de présenter une loi de ce genre à propos de faits révolus ".

10. Venturini 2010, p. 434.

11. On pouvait aussi se demander si le qualificatif d'indemnatus pouvait vraiment s'appliquer à des hommes déclarés ennemis publics par le sénat et cette imprécision laissait à un accusé un moyen de défense (Venturini 1996, p. 252).

12. Cic., Att. 3, 15, 5 : Nam prior lex nos nihil laedebat. Quam si ut est promulgata laudare uoluissemus aut ut erat neglegenda neglegere, nocere omnino nobis non potuisset. Hic mihi primum meum consilium defuit sed etiam obfuit. Caeci, caeci inquam fuimus in uestitu mutando, in populo rogando, quod, nisi nominatim mecum agi coeptum esset, fieri perniciosum fuit.

13. Venturini 2010, p. 437.

14. Moreau 2012, p. 40-41, pour le sénatus-consulte de 62 accordant l'immunité à ceux qui avaient pris à part à l'exécution des complices de Catilina et la technique utilisée par Clodius.

15. Grimal 1967, p. 64-68.

16. La date même de l'affichage de cette mesure, puis de son vote, est difficile à préciser; voir Grimal 1967, p. 70-71 ; Moreau 1987, p. 469-472.

17. Crifò 1961, p. 175-184.

18. Moreau 1987, p. 474 ; Kelly 2006, p. 228.

19. Cic., Dom. 83 : in me cui dies dicta numquam est, qui reus non fui, qui numquam sum a tribuno plebis citatus [...].

20. Garcea 2005, p. 162-170. Sont utilisés aerumna, calamitas, pernicies.

21. Integer (Dom. 9) ; indemnatus (Dom. $9 ; 26 ; 43 ; 77 \ldots$ ) ; on trouve aussi non accusatus (Dom. 26), non citatus.

22. Gruen 1974, p. 246.

23. Moreau 1987, p. 474-475 ; Venturini 2010, p. 439. De plus, Clodius ne semble pas s'embarrasser de légalité républicaine.

24. Cic., Att. 3, 2 ; 3 , 4.

25. Moreau 2005.

26. Moreau 1987, p. 475. Plutarque et Dion Cassius indiquent la distance de 500 milles ; Cicéron, en Att. 3, 4, fait état de quadringenta milia, ce qui peut être une erreur des manuscrits.

27. Shackleton Bailey (éd.) 1965, p. 228.

28. Moreau 2005, p. 208-209.

29. La confiscation, suivie d'une vente aux enchères, concernait tous les biens de Cicéron, y compris les esclaves; Cicéron se préoccupait de leur sort, comme le montre la lettre, datée de la fin avril 58, adressée à Terentia (Fam. 14, 4, 4).

30. Cic., Dom. 50 : [...] pluribus de rebus uno sortitu tulisti [...] ; 53.

31. Cic., Dom. 51 : poena est qui receperit [...] ; Crifò 1961, p. 173 ; Kelly 2006, p. 25-26.

32. Cic., Att. 3, 4 : ne et Sicca apud quem eram periret ; Fam. 14, 4, 2 : Nos Brundisii apud M. Laenium Flaccum dies XIII fuimus, uirum optimum qui periculum fortunarum et capitis sui prae mea salute neglexit neque legis improbissimae poena deductus est quo minus hospitii et amicitiae ius officiumque praestaret.

33. Cic., Dom. 47 : Quid tulit legum scriptor peritus et callidus? [...] Non tulit ut interdicatur. Quid ergo? ut interdictum sit. 
34. Cic., Dom. 47 : quod factum non est, ut sit factum ferri ad populum aut uerbis ullis sanciri aut suffragiis confirmari potest? Voir Moreau 1987, p. 491 ; Kelly 2006, p. 230-231.

35. Moreau 1987, p. 472-473, souligne l'originalité de cet exposé des motifs : "phénomène sans parallèle dans les textes de lois conservés ».

36. Cic., Pis. 72 : QVOD VINDICARIT.

37. Cic., Dom. 50 : QVOD M. TVLLVS FALSVM SENATVS CONSVLTVM RETTVLERIT.

38. Cic., Sul. 40, pour la séance du 3 décembre ; Plu., Cat. Mi. 23, 3-4, pour la séance du 5 décembre. 39. Venturini 1990, p. 268-269; Venturini 2010, p. 442, voit dans cette déclaration un « compromis politique » qui laisse dans le vague le problème du sénatus-consulte ultime et de sa légitimité.

40. Gel., 10, 20, 1 : Quaeri audio, quid «lex» sit, quid " plebisscitum », quid "rogatio ", quid "priuilegium ». 2. Ateius Capito, publici priuatique iuris peritissimus, quid «lex» esset, hisce uerbis definiuit: "Lex» inquit "est generale iussum populi aut plebis rogante magistratu». 3. Ea definitio si probe facta est, neque de imperio $\mathrm{Cn}$. Pompei neque de reditu M. Ciceronis neque de caede P. Clodi quaestio neque alia id genus populi plebisue iussa "lèges" uocari possunt. 4. Non sunt enim generalia iussa neque de uniuersis ciuibus, sed de singulis concepta; quocirca " priuilegia » potius uocari debent, quia ueteres « priua » dixerunt, quae nos « singula » dicimus.

41. Voir Ducos 1984, p. 59-64 ; Cic., Leg. 3, 44 : In priuatos homines leges ferri noluerunt, id est enim priuilegium : quo quid est iniustius, cum legis haec uis sit, <ut sit> scitum et iussum in omnis? Ferri de singulis nisi centuriatis comitiis noluerunt. Voir Venturini 1990 et aussi Humbert 1996.

42. Cic., Att. 3, 15, 5 : Quod te cum Culleone scribis de priuilegio locutum, est aliquid.

43. Cic., Leg. 3, 45 : Quo uerius in causa nostra uir magni ingenii summaque prudentia L. Cotta dicebat nihil omnino actum esse de nobis [...]. Quocirca nihil nobis opus esse lege de quibus omnino nihil actum esset legibus. Cf. Cic., Dom. 68, où est évoquée l'intervention de L. Cotta au sénat, le jour des calendes de janvier 57 ; Sest. 73 où Cicéron rapporte à peu près dans les mêmes termes les paroles de L. Cotta.

44. Cic., Att. 3, 15, 5 : Quod te cum Culleone scribis de priuilegio locutum, est aliquid, sed multo est melius abrogari.

45. Cic., Att. 3, 12, 1 : caput rogationis proponi scribis qua re in senatu dici nihil liceat ; 3, 15, $6:$ Ast tute scripsisti ad me quoddam caput legis Clodium in curiae poste fixisse, NE REFERRI NEVE DICI LICERET; 3, 23, 2 : Scis enim Clodium sanxisse ut uix aut omnino non posset nec per senatum nec per populum infirmari sua lex. Cf. Red. Sen. 8: cum praeclarum caput recitaret ne quis ad uos referret, ne quis decerneret, ne disputaret, ne loqueretur, ne pedibus iret, ne scribendo adesset [...]. Voir l'analyse de Moreau 1989.

46. Cic., Att. 3, 23, 2. Selon Cicéron, ce qui manque dans cette proposition comme dans d'autres, c'est la restitution des biens confisqués ou détruits (Att. 3, 20, 3 ; Fam. 14, 2, 3).

47. Cic., Att. 3, 23, 2 : Alterum caput est tralaticium de impunitate, SI QVID CONTRA ALIAS LEGES EIVS LEGIS ERGO FACTVM SIT. Moreau 1989, p.162-163, s'interroge sur l'exactitude de la citation cicéronienne et conclut qu'il s'agit d'un résumé et non d' « une citation fidèle de l'article d'impunité ».

48. Moreau 1989, p. 158-159.

49. Cic., Att. 3, 23, 2 : Sed uides numquam esse obseruatas sanctiones earum legum quae abrogarentur.

50. Cic., Att. 3, 23, 4 : Tamen in lege nulla esse eius modi caput te non fallit, quod si opus esset, omnes in abrogando uterentur.

51. Le sens du verbe obrogare est discuté: abrogation expresse (Moreau 1989, p.171) ou abrogation tacite, par une loi contraire (cf. P. Festus, 203 L. : legis prioris infirmandae causa legem aliam facere).

52. Marouzeau 1959.

53. Moreau 1989 en montre les différents modèles attestés dans d'autres lois.

54. Mommsen 1892, p. 333 et n. 3.

55. Moreau 1989, p. 154 ; personne ne s'attendait d'ailleurs à un vote favorable. 
56. Cic., Att. 3, 20, 2 : nihil malo quam domum.

57. Cic., Fam. 14, 2, 3 : ego uero tum denique mihi uidebor restitutus, si illa nobis erit restituta.

58. Cic., Att. 1, 3, 6 ; Off. 1, 138-139.

59. Cic., Dom. 106 ; Moreau 1987, p. 479-480.

60. Cic., Att. 4, $1,7$.

61. Cic., Att. 4, 2, 2-3.

62. Cic., Dom. 51 : praeterquam quod ne id quidem per legem Liciniam, ut tibi curationem ferres, facere potuisti. Cf. Dom. 106 : tuleram ut mihi liceret.

63. Cic., Dom. 127 : Video enim esse legem ueterem tribuniciam, quae uetet iniussu plebis, aeris, terram, aram consecrari. Il s'agit de la lex Papiria

64. Cic., Dom. 140.

65. Berg 1997.

\section{RÉSUMÉS}

Nous proposons dans cet article quelques analyses sur les problèmes de droit présents dans les lettres de Cicéron des années 58-57 av. J.-C. Le consulaire exilé se montre en effet attentif aux questions juridiques liées à sa situation personnelle.

This article deals with the law issues wich are present in the

\section{INDEX}

Keywords : Clodius, exil, Cicero's letters, De domo, Lex Clodia de capite civis Romani, Lex Clodia de exilio Ciceronis

nomsmotscles Appien, Ateius Capito, Aulu-Gelle, Cicéron, Dion Cassius, Festus grammaticus, Plutarque, Velleius Paterculus

Mots-clés : Clodius, exil, lettres de Cicéron, De domo, Lex Clodia de capite civis Romani, Lex Clodia de exilio Ciceronis

\section{AUTEUR}

\section{MICHĖLE DUCOS}

Université Paris-Sorbonne 